\title{
К вопросу о концептуальных основаниях функционирования программного обеспечения для репрезентации делиберативной аргументации
}

\author{
Е.Н. Лисанюк, Д.Е. Прокудин \\ Санкт-Петербургский государственный университет \\ e.lisanuk@spbu.ru, d.prokudin@spbu.ru
}

\section{Аннотация}

Делиберативная аргументация находит широкое применение в различных областях деятельности человека. В современном информационном обществе применение методов делиберативной аргументации связано с разработкой и использованием соответствующего прикладного программного обеспечения, которое предназначено для визуализации и моделирования интеллектуальной деятельности по решению различного рода практических задач, а также аргументации. При этом в различном программном обеспечении, предназначенном для моделирования и репрезентации аргументации, явно или неявно заложены её концептуальные основания аргументации. В настоящем исследовании на основе выявления программного обеспечения, предназначенного для моделирования делиберативной аргументации, анализа его назначения и основных функций определяются концептуальные основания их функционирования, что является начальным этапом для формулирования корпуса критериев оценки данного программного обеспечения и его последующей классификации.

Ключевые слова: делиберативная аргументация, концептуальные основания, программное обеспечение, моделирование, репрезентация

Библиографическая ссылка: Лисанюк Е.Н., Прокудин Д.Е. К вопросу о концептуальных основаниях функционирования программного обеспечения для репрезентации делиберативной аргументации // Информационное общество: образование, наука, культура и технологии будущего. Выпуск 4 (Труды XXIII Международной объединенной научной конференции «Интернет и современное общество», IMS-2020 (сборник научных статей). - СПб: Университет ИТМО, 2020. С. 34-41. DOI: $10.17586 / 2587-8557-2020-4-34-41$

\section{Введение}

Делиберативная аргументация - это разновидность аргументации о действиях, в отличие от теоретической аргументации, в центре которой находится обоснование истинности предложения. Делиберативная аргументация фокусируется на обосновании по поводу того, что нужно делать или как поступать в той или иной ситуации. Помимо действий, делиберативная аргументация касается также норм и ценностей, выступающих атомарными элементами делиберативного обоснования, поэтому она находит широкое применение в различных областях практической деятельности человека, включая политическую, правовую, социальную и повседневную жизнь. В контексте создания ПО для репрезентации делиберативной аргументации ее главное отличие от теоретической состоит в двух аспектах, техническом и концептуальном. Техническое отличие связано с тем, что для делиберативной аргументации нужны более широкие выразительные возможности, чтобы помимо описательных предложений учитывать намерения, цели, 
нормы, ценности и т.п. [3] Концептуальное отличие заключается в разграничении между аргументацией как конкуренции обоснований, которая относится к моделированию рассуждений вообще, манипуляциями с базами знаний и информацией, составляющими когнитивное направление в информатике, и принятием решений как стратегии и тактики поведения, относящимся к сфере психологии и управления. Провести отбор и классификацию ПО с учетом этих трех аспектов - конечная цель нашего исследования. На его начальном пилотном этапе мы провели предварительный отбор ПО для репрезентации аргументации и рассуждений в целом, отделив от него ПО, применяемое для манипуляций с базами знаний и информацией; на втором этапе мы ставим задачу отбора и классификации ПО для моделирования делиберативной аргументации, чтобы в дальнейшем инкорпорировать ее в итоговую классификацию. Здесь речь пойдет об общих подходах на втором этапе.

В современном информационном обществе моделирование делиберативной аргументации связано с разработкой и использованием соответствующего прикладного программного обеспечения, которое предназначено для визуализации интеллектуальной деятельности по решению различного рода практических задач, в том числе и аргументации. Это означает, что большинство ПО не проводит упомянутого выше концептуального разграничения. Методы поддержки интеллектуальной деятельности с элементами делиберации с использованием информационно-коммуникационных технологий, направленные на принятие решений, находят своё применение в различных областях человеческой деятельности: медицине [15], публичной политике и электронной демократии $[8,12]$, научной или академической аргументации (включая технические, медицинские и гуманитарные науки) [17], образовании, бизнесе и других областях $[19,20]$.

При этом в различном программном обеспечении, предназначенном для моделирования и репрезентации аргументации, явно или неявно заложены разные концептуальные основания аргументации. В настоящем исследовании на основе выявления программного обеспечения, предназначенного для моделирования делиберативной аргументации, анализа его назначения и основных функций определяются концептуальные основания их функционирования, что является начальным этапом для формулирования корпуса критериев оценки данного программного обеспечения и его последующей комплексной классификации.

Разработка такой классификации преследует следующие цели:

- выработка предложений для решения актуальной проблемы, состоящей в теоретическом разрыве между концепциями аргументации, сформулированными в результате ее научного исследования, и концепциями, явно или неявно подразумеваемыми в ряде программных систем и приложений, предназначенных для ее моделирования и репрезентации. Большинство таких систем и приложений носят дескриптивный характер: они ограничиваются визуализацией аргументативных диалогов (споров) и не предлагают механизмов для их решений, поэтому важно установить, какие именно аспекты делиберации визуализирует то или иное ПО;

- установление концептуальных границ между тремя подходами: собственно моделированием аргументации - разновидностью интеллектуальной познавательной деятельности, нацеленной установить состоятельность или убедительность мнений сторон по какому-либо вопросу; визуализацией критических и делиберативных рассуждений - прикладными методиками картирования рассуждений и майндмэппингом - наборов графических способов представления информации, включая рассуждения, при помощи ассоциативных диаграмм;

- предоставить академическому, исследовательскому и образовательному сообществу инструмент эффективного выбора программных систем и приложений для использования в исследовательской и образовательной деятельности, основанной на применении методов делиберативной аргументации; 
- в прикладном аспекте оказать методическую поддержку для формулировки рекомендаций для создания отечественных программных систем и приложений для моделирования аргументации.

\section{1. Выявление концептуальных оснований программного обеспечения для делиберативных рассуждений и аргументации}

В результате проведённого ранее пилотного исследования был произведён отбор программного обеспечения, предназначенного для моделирования, анализа и обучения навыкам аргументации и критического мышления [4, 5]. Отобранное программное обеспечение было разбито на несколько основных категорий в соответствии с основным назначением:

- моделирование аргументации;

- визуализация критических и делиберативные рассуждений;

- майндмэппинг.

При этом некоторые программные системы и приложения можно соотнести с несколькими из выделенных категорий.

На настоящем этапе исследования отобран и рассмотрен ещё ряд программных систем и приложений. На отобранном массиве для выявления концептуальных основ функционирования рассматриваемых программных продуктов были применены различные методы:

- некоторое программное обеспечение уже достаточно давно используется нами в учебном процессе и концептуальные основания выявлены в практике его применения;

- ряд программных систем и приложений используются в практической деятельности и их характеристики описаны в учебно-методической и научной литературе;

- разработчики отдельных решений сами описывают основные принципы их функционирования;

- концептуальные особенности функционирования некоторых представителей подобного программного обеспечения исследованы и представлены в соответствующих научных публикациях.

Например, концептуальным фундаментом программных приложений Carneades и Rationale, используемых нами в учебном процессе, считается модель аргументации Ст. Тулмина, которая изначально трактовалась исследователями в нормативнофункциональном или нормативно-структурном ключе. Концепции аргументации, имплементированные в Carneades и Rationale, различны и обе являются дескриптивными. B Carneades модель Ст. Тулмина дополнена разными видами отменяемых рассуждений, а в Rationale - качественными оценками аргументов на основе установления их содержательных источников (данные, статистика, экспертные мнения и т.п.), а также шаблонами порождения аргументации в тексте. Недавно разработчики Rationale предложили новое приложение для моделирования принятия решений b'cisive, в основе которого лежит концепция делиберативного протокола [7]. Разработчики b'cisive позиционируют его для визуализации и делиберативной аргументации, и принятия решений, сознательно отказываясь проводить разграничение между ними.

Некоторые программы уже не поддерживаются и не развиваются. К ним, например, относится программное приложение Araucaria, классифицированное нами для использования при моделировании аргументации и делиберативных рассуждений (с 2006 года разработчики его не развивают и не поддерживают). На его смену пришло вебориентированное приложение OVA (http://ova.arg-tech.org), предназначенное для конструирования аргументационных карт с целью анализа и моделирования аргументации в тексте. А в этом приложении уже реализовано разграничение между разновидностями аргументов по качеству и функциям. 
В нём для пользователя доступны различные наборы схем аргументации (например, Walton presumptive inference, Rutgers SALTS, Cornell, Dundee illocutionary, Second order illocutionary, Basic conflict, Extended Conflict, Deductive inference), которые используются для картирования текстов из различных предметных областей, включающих дедуктивные и разнообразные недедуктивные аргументы.

Исходя из этого, можно заключить, что, несмотря на базировании одних и тех же концепций аргументации, приложения отличаются по степени их реализации. Например, для использования ПО Rationale пользователю не требуется никаких предварительных теоретических знаний об анализе и строении аргументации. В нём реализована возможность для порождения текстов, содержащих аргументацию в духе дизайнмышления, а также возможность многофакторной оценки эффективности аргументации. Две последние опции не предусмотрены. Визуализации аргументации в Rationale приближена к интуитивному ее картированию средствами уже имеющихся теорий, отраженных в стандартных учебниках по аргументации. В отличие от этого, в ПО OVA на пользователя ложится дополнительная задача по выбору схему аргументации и конструированию карты следуя заданным теоретическим основанием, хорошо известным самому пользователю.

Также в рамках исследования нами выделяются в отдельную группу программные системы и платформы, использующиеся для поддержки делиберативной демократии (например, DemocracyOS, Democracy 2.1, Loomio, OpaVote, Delib, Decidim) [6, 11], в концепции которой принятие политических решений, формулировка политической повестки и рассмотрение спорных вопросов основывается на делиберативном общественном мнении. В основном эти инструменты на сегодняшний день представляют собой социальные интернет-площадки или форумы с инструментами, которые реализующие опросы, обмен мнениями, дебаты и дискуссии, статистический анализ которых и визуальное представление его результатов, нацелены на принятие решений в сфере государственного и муниципального управления.

При этом аналитическая функция отводится при реализации такого подхода человеку. Хотя апологеты делиберативной демократии уже задумываются о возможности применения систем искусственного интеллекта (AI) и технологий обработки естественного языка (NLP) для анализа общественного дискурса и принятия решений [16]. В связи с этим в настоящее время нельзя выделить какие-либо концептуальные основания аргументации, имплементированные в подобного рода программные системы и платформы.

Мы предлагаем две предварительные классификации программных систем и приложений, а также теоретических описаний, которые могут быть использованы для создания подобного ПО. Сначала мы поделили их на две группы в зависимости от того, представляют они собой дескриптивные формализмы (протоколы, онтологии), визуализирующие рассуждения пользователей, или нормативные, моделирующие то, какие заключения нужно (рекомендуется) вывести или какие решения принять Таблица 1. Затем мы выделили две группы теоретических концепций, заложенные разработчиками в соответствующее ПО, в зависимости от того, поддерживает оно визуализацию модифицируемых (отменяемых) рассуждений или нет - Таблица 2.

Как видно из этих двух классификаций, выбранные нами критерии дают независимые группы, и использование этих критериев для создания обобщенной классификации нецелесообразно.

Помимо изученных нами и описанных программных решений существуют другие программные системы и платформы, в которых имплементированы механизмы делиберации, аргументации и поддержки интеллектуальной деятельности. Однако, они описаны только в научной литературе (например, ProGraph, ConArg2), отсутствуют ссылки как на сайты разработчиков, так и на само программное обеспечение. 
Таблица 1. Дескриптивные и нормативные приложения

\begin{tabular}{|l|l|}
\hline \multicolumn{1}{|c|}{ Протоколы $\backslash$ онтологии $\backslash$ платформы } & \multicolumn{1}{|c|}{ Нормативные формализмы } \\
\hline $\begin{array}{l}\text { Приложения для визуализации аргументации } \\
\text { (Carneades, Rationale, b'cisive) [10] }\end{array}$ & $\begin{array}{l}\text { моделирование отменяемых рассуждений } \\
\text { (defeasible reasoning) (DeLP, DefLog) }\end{array}$ \\
\hline $\begin{array}{l}\text { Платформа для обсуждений и неформальных } \\
\text { диалогов (D-BAS) [13] }\end{array}$ & $\begin{array}{l}\text { Моделирование аргументации при помощи } \\
\text { систем искусственного интеллекта (ArgTools, } \\
\text { Dung-O-Matic) [5. 18] }\end{array}$ \\
\hline $\begin{array}{l}\text { IBIS (Issue-Based Information System) - } \\
\text { плаформа для совместного выявления и } \\
\text { решения проблем (QuestMap, Compendium) } \\
\text { [14] }\end{array}$ & $\begin{array}{l}\text { Моделирование вероятностных умозаключений } \\
\text { с модифицируемыми допущениями (PITA) }\end{array}$ \\
\hline $\begin{array}{l}\text { Онтология аргументации для отдельных видов } \\
\text { рассуждений [2] }\end{array}$ & $\begin{array}{l}\text { Моделирование когнитивных рассуждений для } \\
\text { рациональных агентов (OSCAR) [18] }\end{array}$ \\
\hline Протокол аргументации о действиях АТR [7] & $\begin{array}{l}\text { Моделирование аргументации в диалоге } \\
\text { посредством игры для двух агентов (Convince } \\
\text { Ме) }\end{array}$ \\
\hline Система правдоподобной аргументации [1] & \multicolumn{2}{|l}{} \\
\hline
\end{tabular}

Таблица 2. Приложения с опциями для модифицируемых рассуждений и без.

\begin{tabular}{|c|c|}
\hline Немодифицируемые (монотонные) & Модифицируемые (немонотонные) \\
\hline $\begin{array}{l}\text { Дескриптивная модель аргументации Ст. } \\
\text { Тулмина (Carneades, Rationale, b'cisive) [10] }\end{array}$ & $\begin{array}{l}\text { моделирование отменяемых рассуждений } \\
\text { (defeasible reasoning) (DeLP, DefLog) }\end{array}$ \\
\hline $\begin{array}{l}\text { Формализованная модель проведения } \\
\text { неформальных диалогов (неявная } \\
\text { аргументация) (D-BAS) [13] }\end{array}$ & $\begin{array}{l}\text { Моделирование аргументации при помощи } \\
\text { систем искусственного интеллекта (ArgTools, } \\
\text { Dung-O-Matic) }[9,18]\end{array}$ \\
\hline $\begin{array}{l}\text { IBIS (Issue-Based Information System) - подход } \\
\text { объяснительной аргументации, разработанный } \\
\text { В. Кунцем и Х. Риттелем и предназначенный } \\
\text { для совместного выявления и решения } \\
\text { проблем (QuestMap, Compendium) [14] }\end{array}$ & $\begin{array}{l}\text { Моделирование вероятностных умозаключений } \\
\text { с модифицируемыми допущениями (РITA) }\end{array}$ \\
\hline $\begin{array}{l}\text { Онтология аргументации для отдельных видов } \\
\text { рассуждений [2] }\end{array}$ & $\begin{array}{l}\text { Моделирование когнитивных рассуждений для } \\
\text { рациональных агентов (OSCAR) [18] }\end{array}$ \\
\hline \multirow[t]{2}{*}{$\begin{array}{l}\text { Моделирование аргументации в диалоге } \\
\text { посредством игры для двух агентов (Convince } \\
\text { Ме) }\end{array}$} & Протокол аргументации о действиях ATR [7] \\
\hline & Система правдоподобной аргументации [1] \\
\hline
\end{tabular}

Поэтому, скорее всего, они представляют собой либо продолжающиеся разработки, либо тестовые варианты, что не позволяет изучить их возможности и описать основные характеристики, в том числе и концептуальные основы их построения.

\section{Заключение}

На текущем этапе исследования выявлены два основания разделения программного обеспечения - по основанию дискриптивности/нормативности и по модифицируемости рассуждений, что ляжет в основу разрабатываемой классификации данного ПО.

В дальнейшем в целях разработки комплексной классификации программном обеспечении, предназначенном для моделирования и репрезентации аргументации, предполагается выделить основные концептуальные основания, которые позволят сформировать укрупнённые группы программного обеспечения, а различия в реализации этих концепций в ПО рассматривать как функциональные особенности и выделить их в отдельную характеристику, которая позволит пользователям более рационально подходить к выбору программных систем и приложений для применения в решаемых ими задачах. 
Работа выполнена при поддержке Российского фонда фундаментальных исследований, проект 20-011-00485-а.

\section{Литература}

[1] Вагин В.Н., Моросин О.Н. Программная реализация системы аргументации со степенями обоснования// Программные продукты и системы. 2015. № 1. С. 21-27. DOI: 10.15827/0236-235X.109.021-027.

[2] Загорулько, Ю.А. Моделирование аргументации в научно-популярном дискурсе с использованием онтологий / Ю.А. Загорулько, Н.О. Гаранина, О.И. Боровикова, О.А. Доманов // Онтология проектирования. 2019. Т. 9, №4(34). С.496-509. DOI: 10.18287/2223-9537-2019-9-4-496-509.

[3] Лисанюк Е.Н. Аргументация и убеждение. СПб, Наука. 2015.

[4] Лисанюк Е.Н., Прокудин Д.Е. Модели обучения аргументации с использованием программного обеспечения // Логико-философские штудии. 2016. Т. 13, № 2. C. $217-218$.

[5] Лисанюк Е.Н., Прокудин Д.Е. Программное обеспечение для моделирования аргументации: классификация и сравнительный анализ // Интернет и современное общество: сборник тезисов докладов [Электронный ресурс] / Труды XX Международной объединенной научной конференции «Интернет и современное общество» (IMS- 2017), Санкт-Петербург, 21-23 июня 2017 г. Электрон, дан. СПб: Университет ИТМО, 2017. С. 11-13. http://ojs.ifmo.ru/index.php/IMS/article/view/516 (дата обращения: 22.12.2019).

[6] Aragón P., Kaltenbrunner A., Calleja-López A., Pereira A., Monterde A., Barandiaran X. E., Gómez V. Deliberative Platform Design: The Case Study of the Online Discussions in Decidim Barcelona. 2017. ArXiv:1707.06526.

[7] Atkinson K., Bench-Capon T. Practical reasoning as presumptive argumentation using action based alternating transition systems // Artificial Intelligence. 2007. №. 171. P. 855-874.

[8] Davies T., Chandler R. Online deliberation design: Choices, criteria, and evidence // Democracy in motion: Evaluating the practice and impact of deliberative civic engagement / Nabatchi T., Weiksner M., Gastil J., Leighninger M. (eds.). Oxford: Oxford univ. press, 2013. P. 103-131.

[9] Dung P. On the acceptability of arguments and its fundamental role in nonmonotonic reasoning, logic programming, and n-person games // Artificial Intelligence. 1995. №. 77. P. 321-357.

[10] Gordon T.F. An Overview of the Carneades Argumentation Support System // Dialectics, Dialogue and Argumentation. An Examination of Douglas Waltons Theories of Reasoning / Tindale, C.W., Reed, C. (eds.). 2010. P. 145-156.

[11] Grassle S. Digital tools for participatory democracy // GovLab Blog. 2015. 5 марта. $\mathrm{http} / /$ thegovlab.org/digital-tools-for-participatory-democracy (дата обращения: 19.12.2019).

[12]Klein M., Iandoli L. Supporting Collaborative Deliberation Using a Large-Scale Argumentation System: The MIT Collaboratorium // Proceedings of the Eleventh Directions and Implications of Advanced Computing Symposium and the Third International Conference on Online Deliberation (DIAC_2008/OD 2008), Berkeley, California. 2008. P. 5-12. DOI:10.2139/ssrn.1099082.

[13] Krauthoff T., Meter C., Baurmann M., Betz G., Mauve M. D-BAS-A Dialog-Based Online Argumentation System // Computational Models of Argument: Proceedings of COMMA 2018 305. 2018. P. 325-336. DOI: 10.3233/978-1-61499-906-5-325.

[14] Kunz W., Rittel H. Issues as elements of information systems. Working Paper No. 131, Institute of Urban and Regional Development, Univ. of California, Berkeley, Calif., 1970. 
[15] Mahadevan, N., A. Dubey, D. Balasubramanian, Karsai G. Deliberative, search-based mitigation strategies for model-based software health management // Innovations in Systems and Software Engineering. Springer London. 2013. P. 1-26.

[16] Mejia M. How can digital tools support deliberation? Join the conversation! // Medium. 2020. 10 апреля. URL: https://medium.com/participo/how-can-digital-tools-supportdeliberation-join-the-conversation-2f0622bda62a (дата обращения: 28.12.2019).

[17] Online Deliberation: Design, Research, and Practice. Davies T., Gangadharan S.P. (eds.). Stanford, CSLI Publications. 2009.

[18] Pollock J. Cognitive Carpentry. A Blueprint for How to Build a Person. MIT Press, 1995.

[19] Schneider D., Voigt C., Betz G. Argunet: A software tool for collaborative argumentation analysis and research // 7th Workshop on Computational Models of Natural Argument (CMNA 2007), Hyderabad, India. 2007.

[20] Visualizing Argumentation. Computer Supported Cooperative Work. Kirschner, P. A., Buckingham Shum, S. J., Carr, C. S. (Eds.). Springer, London, 2003. DOI:10.1007/978-14471-0037-9.

\title{
Study of Conceptual Bases of Software Functioning for the Representation of Deliberative Argumentation
}

\author{
E.N. Lisanyuk, D.E. Prokudin
}

\section{St Petersburg State University}

Deliberative reasoning is widely used in various fields of human activity. In the modern information society, the use of methods of deliberative argumentation is associated with the development and use of appropriate application software, which is intended for visualization and modeling of intellectual activity to solve various types of practical problems, as well as argumentation. At the same time, various software designed for modeling and representation of argumentation explicitly or implicitly provides its conceptual basis for argumentation. In this study, based on the identification of software intended for the simulation of deliberative reasoning, analysis of its purpose and main functions, the conceptual foundations of their functioning are determined, which is the initial stage for the formulation of a body of criteria for evaluating this software and its subsequent classification.

Keywords: deliberative reasoning, conceptual bases, software, modeling, representation

Reference for citation: E.N. Lisanyuk, D.E. Prokudin Study of Conceptual Bases of Software Functioning for the Representation of Deliberative Argumentation // Information Society: Education, Science, Culture and Technologies of the Future. Vol. 4 (Proceedings of the XXIII International Joint Scientific Conference «Internet and Modern Society», IMS-2020, St. Petersburg, June 17-20, 2020). - St. Petersburg: ITMO University, 2020. P. 34 - 41. DOI: $10.17586 / 2587-8557-2020-4-34-41$

\section{References}

[1] Vagin V.N., Morosin O.N. Software implementation of an argumentation system withjustification degrees // Software \& Systems. 2015. № 1. P. 21 -27. DOI: 10.15827/0236235X.109.021-027.

[2] Zagorulko Yu.A., Garanina N.O., Borovikova O.I., Domanov O.A. Argumentation modeling in popular science discourse using ontologies // Ontology of Designing. 2019. T. 9. №4(34). C.496-509. DOI: 10.18287/2223-9537-2019-9-4-496-509.

[3] Lisanyuk E.N. Argumentacija i ubezhdenie. SPb, Nauka. 2015. 
[4] Lisanyuk E.N., Prokudin D.E. Modeli obuchenija argumentacii $\mathrm{s}$ ispol'zovaniem programmnogo obespechenija // Logiko-filosofskie shtudii. 2016. V. 13. № 2. P. 217-218.

[5] Lisanyuk E.N., Prokudin D.E. Classifying software for argumentation modelling // Internet $\mathrm{i}$ sovremennoe obshhestvo: sbornik tezisov dokladov / Trudy XX Mezhdunarodnoj ob'edinennoj nauchnoj konferencii «Internet and Modern Society» (IMS-2017), St.Peterburg, June, 21-23, 2017, SPb., ITMO University, $2017 . \quad$ P. 11-13. http://ojs.ifmo.ru/index.php/IMS/article/view/516.

[6] Aragón P., Kaltenbrunner A., Calleja-López A., Pereira A., Monterde A., Barandiaran X. E., Gómez V. Deliberative Platform Design: The Case Study of the Online Discussions in Decidim Barcelona. 2017. ArXiv:1707.06526.

[7] Atkinson K., Bench-Capon T., Practical reasoning as presumptive argumentation using action based alternating transition systems // Artificial Intelligence. 2007. № 171. P. 855-874.

[8] Davies T., Chandler R. Online deliberation design: Choices, criteria, and evidence // Democracy in motion: Evaluating the practice and impact of deliberative civic engagement / Nabatchi T., Weiksner M., Gastil J., Leighninger M. (eds.). Oxford: Oxford univ. press, 2013. P. 103-131.

[9] Dung P. On the acceptability of arguments and its fundamental role in nonmonotonic reasoning, logic programming, and n-person games // Artificial Intelligence, 1995. № 77. P. 321-357.

[10] Gordon T.F. An Overview of the Carneades Argumentation Support System // Dialectics, Dialogue and Argumentation. An Examination of Douglas Waltons Theories of Reasoning / Tindale, C.W., Reed, C. (eds.). 2010. P. 145-156.

[11] Grassle S. Digital tools for participatory democracy // GovLab Blog. 2015. 5 марта. http://thegovlab.org/digital-tools-for-participatory-democracy.

[12]Klein M., Iandoli L. Supporting Collaborative Deliberation Using a Large-Scale Argumentation System: The MIT Collaboratorium // Proceedings of the Eleventh Directions and Implications of Advanced Computing Symposium and the Third International Conference on Online Deliberation (DIAC_2008/OD 2008), Berkeley, California. 2008. P. 5-12.

[13] Krauthoff T., Meter C., Baurmann M., Betz G., Mauve M. D-BAS-A Dialog-Based Online Argumentation System // Computational Models of Argument: Proceedings of COMMA 2018 305. 2018. P. 325-336. DOI: 10.3233/978-1-61499-906-5-325.

[14]Kunz W., Rittel H. Issues as elements of information systems. Working Paper № 131, Institute of Urban and Regional Development, Univ. of California, Berkeley, Calif., 1970.

[15] Mahadevan, N., A. Dubey, D. Balasubramanian, Karsai G. Deliberative, search-based mitigation strategies for model-based software health management // Innovations in Systems and Software Engineering. Springer London, 2013. P. 1-26.

[16] Mejia M. How can digital tools support deliberation? Join the conversation! // Medium. 2020. 10 апреля. https://medium.com/participo/how-can-digital-tools-support-deliberationjoin-the-conversation-2f0622bda62a.

[17] Online Deliberation: Design, Research, and Practice. Davies T., Gangadharan S.P. (eds.). Stanford, CSLI Publications. 2009.

[18] Pollock J. Cognitive Carpentry. A Blueprint for How to Build a Person. MIT Press, 1995.

[19] Schneider D., Voigt C., Betz G. Argunet: A software tool for collaborative argumentation analysis and research // 7th Workshop on Computational Models of Natural Argument (CMNA 2007), Hyderabad, India. 2007.

[20] Visualizing Argumentation. Computer Supported Cooperative Work. Kirschner, P. A., Buckingham Shum, S. J., Carr, C. S. (Eds.). Springer, London, 2003. DOI:10.1007/978-14471-0037-9. 